\title{
Salmonella Osteomyelitis of the Proximal Tibia in a Previously Healthy Adolescent: A Case Report
}

Georgios Vynichakis ${ }^{1}$, Michail Chandrinos ${ }^{2}$, Stavros Angelis ${ }^{3}$, Elefterios Bogris ${ }^{1}$, John N. Michelarakis ${ }^{4}$

1. Orthopaedics, General Hospital of Piraeus Tzaneio, Piraeus, GRC 2. Ortopaedics, General Hospital of Piraeus Tzaneio, Piraeus, GRC 3. Orthopaedics, General Hospital Hellenic Red Cross Korgialenio Benakio, Athens, GRC 4. Orthopaedics, General Children's Hospital “Panagiotis \& Aglaia Kyriakou”, Athens, GRC

Corresponding author: Georgios Vynichakis, vini_gio@windowslive.com

\begin{abstract}
Salmonella osteomyelitis is an uncommon pathological condition. Usually, it is associated with hemoglobinopathies or other underlying disorders. Osteomyelitis due to Salmonella is extremely rare in a previously healthy patient. We present a case of a 12-year-old previously healthy male who suffered Salmonella osteomyelitis of the proximal tibia as well as the diagnostic algorithm and therapy protocol. In our case, Salmonella osteomyelitis was identified by direct inoculation of the sample in blood culture bottles. Traditional cultures were negative. The practice of blood culture bottles seems to be essential for diagnosis, so the appropriate treatment is performed.
\end{abstract}

Categories: Pediatrics, Orthopedics

Keywords: salmonella, adolescent, osteomyelitis, child, tibia, blood culture bottle

\section{Introduction}

Osteomyelitis is uncommon in adolescents and children [1]. A wide range of bacteria could be the cause for osteomyelitis. The most common pathogen is Staphylococcus aureus counting for more than $50 \%$ of all cases. Streptococcus, Escherichia coli, Klebsiella and Proteus are other bacterial pathogens that can cause osteomyelitis [1-2].

Salmonella osteomyelitis is essentially scarce. It counts for $0.8 \%$ of all Salmonella infections and only $0.45 \%$ of all types of osteomyelitis [1-8].

Osteomyelitis due to Salmonella is extremely rare in healthy individuals. Only a few cases have been reported in the literature [1, 3-4]. Usually, Salmonella osteomyelitis is associated with hemoglobinopathies such as sickle-cell disease or thalassemia and it seems to be a significant cause of morbidity and mortality in these individuals [1, 3-8]. Other predisposing factors that have been associated with Salmonella osteomyelitis are the history of trauma or surgery, steroids, malignancy (lymphoma), liver and cardiovascular disease, autoimmune disease (systemic lupus erythematosus), diabetes, chronic

Received 09/03/2019

Review began 09/06/2019 Review ended 09/12/2019 Published 09/16/2019

๑) Copyright 2019

Vynichakis et al. This is an open access article distributed under the terms of the Creative Commons Attribution License CC-BY 3.0., which permits unrestricted use, distribution, and reproduction in any medium, provided the original author and source are credited. granulomatous disease, immunodeficiencies and HIV infection [3-4].

\section{Case Presentation}

A 12-year-old non-ambulatory male was referred to the emergency department of our hospital. He complained about pain in the left knee, fever (up to $40^{\circ} \mathrm{C}$ ), swelling, inability to move and walk properly. Regarding his personal medical history, no trauma or chronic disorder was referred by the patient or the parents. Clinical examination revealed swelling of the knee, stiffness, pain, and restriction of passive movement. Laboratory tests revealed a serum white blood cells (WBC) count of $12700 / \mu$ l (normal: $<10000 / \mu \mathrm{l}$ ), an erythrocyte sedimentation rate (ESR) of $49 \mathrm{~mm}$ per hour (normal: $<25 \mathrm{~mm} / \mathrm{h}$ ) and C-reactive protein (CRP) $55 \mathrm{mg} / \mathrm{L}$ (normal: $<5 \mathrm{mg} / \mathrm{L}$ ) with normal liver function and electrolyte values. Blood cultures were negative. Radiographs revealed lytic lesions in the metaphyseal region of the proximal tibia (Figure 1). Osteomyelitis was suspected. The patient was admitted for monitoring, per os antibiotic therapy with amoxicillin and clavulanic acid and further evaluation. 


\section{Cureus}

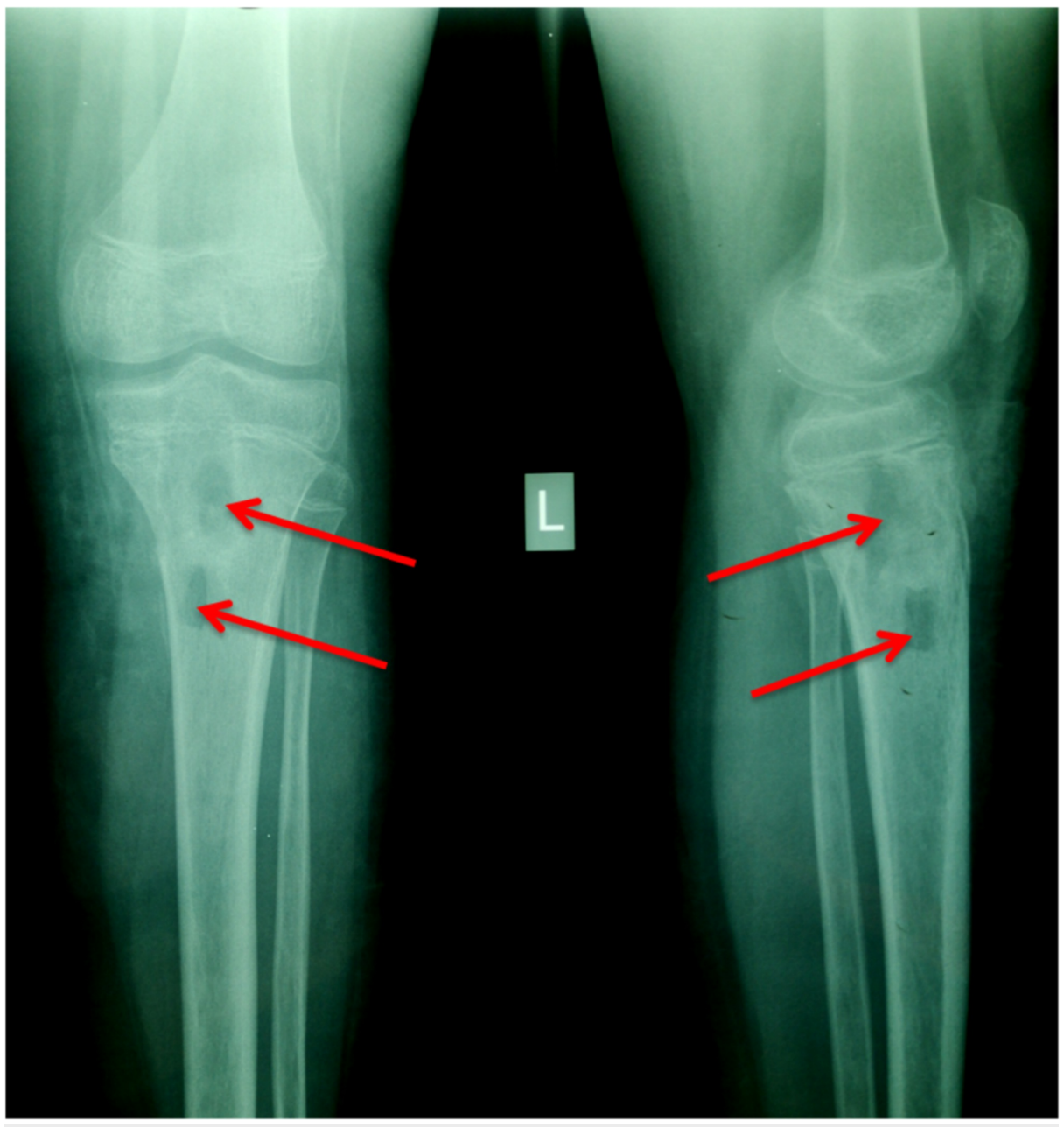

FIGURE 1: Knee radiograph: proximal tibia lytic lesions (red arrows)

Lytic lesions were further evaluated with knee computed tomography (CT) and magnetic resonance imaging (MRI) (Figures 2, 3). The MRI and CT revealed that the lesions extended not only in the metaphyseal region but also in the epiphyseal.

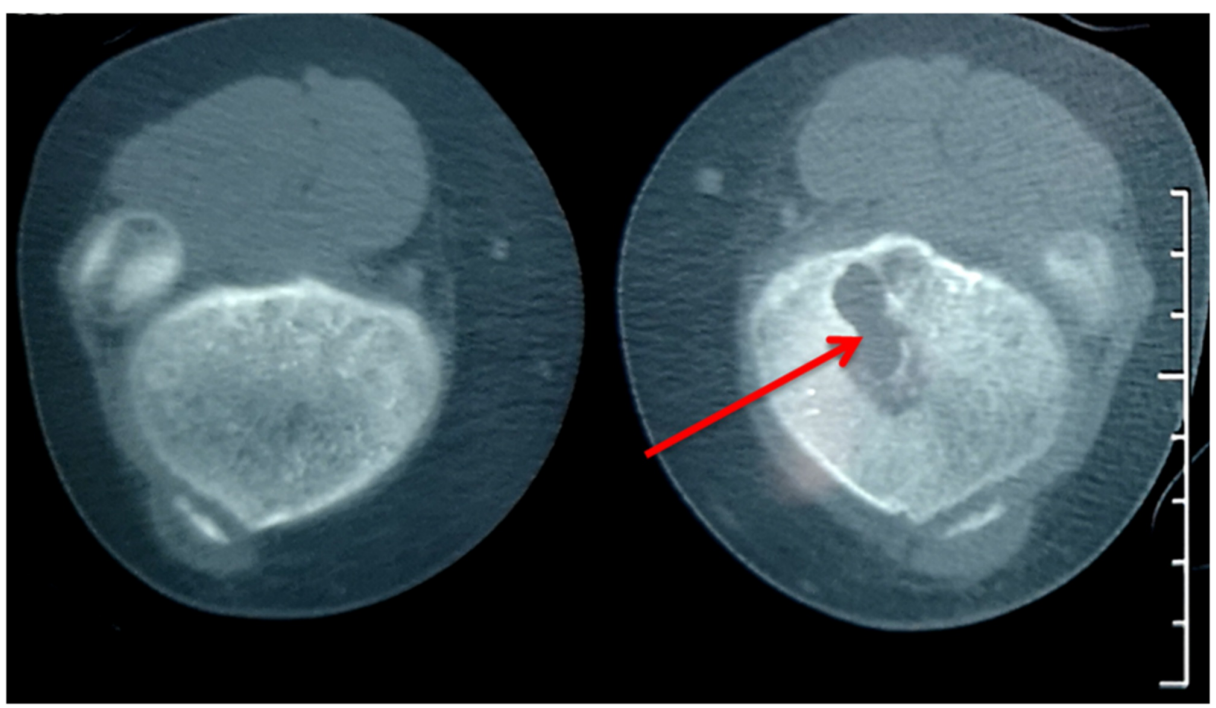

FIGURE 2: Knee CT: lytic lesion in the proximal left tibia (red arrow) 


\section{Cureus}

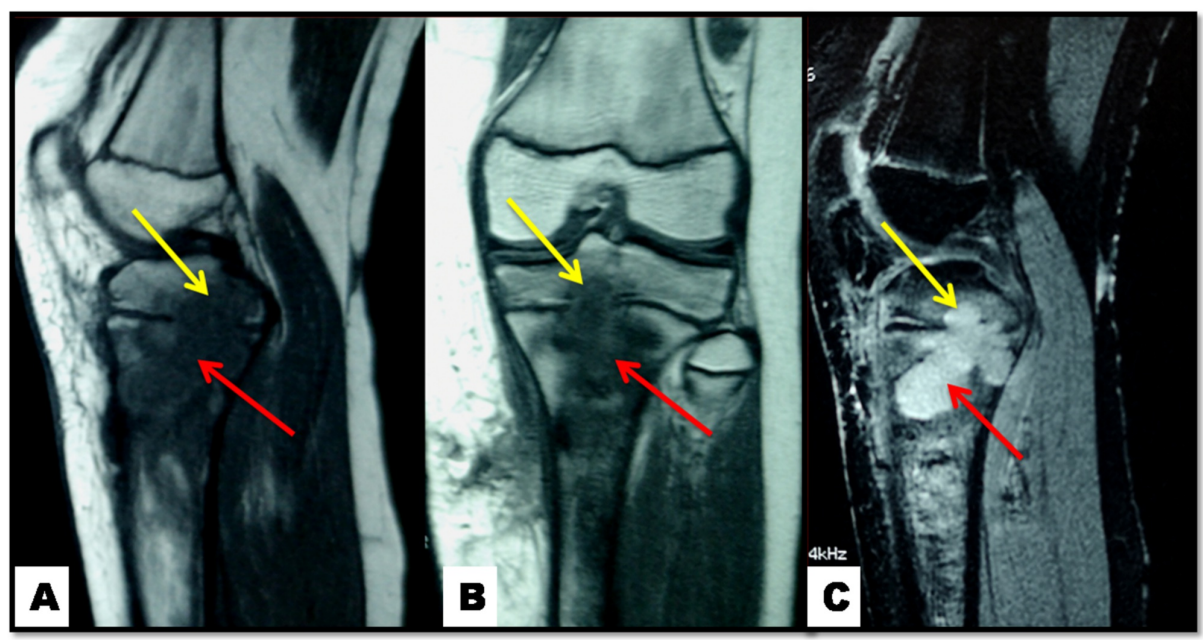

FIGURE 3: Knee MRI: Iytic lesion at metaphyseal (red arrows) and epiphyseal (yellow arrows) regions

A: sagittal in T1-weighted sequence; B: coronal in T1-weighted sequence; C: sagittal T2-weighted sequence

After pediatric infectious diseases specialist's (PIDS) consultation a minimally invasive surgery was decided. Bone and lesion sample for cultures and histological examination was taken and curettage of the lytic lesion was performed. Cultures were negative but histological soft tissue examination revealed inflammation. Intravenous (IV) clindamycin was administered for 12 days. Per os antibiotic treatment (clindamycin) was totally administered for eight weeks.

Three months post-surgery the patient returns experiencing relapse knee swelling and pain. The surgical incision was festering. Laboratory examination revealed WBC count of $8000 / \mu \mathrm{l}$, ESR of $28 \mathrm{~mm}$ per hour and CRP $4 \mathrm{mg} / \mathrm{L}$ with normal liver function and electrolyte values. The child was rehospitalized. After PIDS consultation, IV vancomycin, second knee-MRI and second surgery were decided (Figure 4).

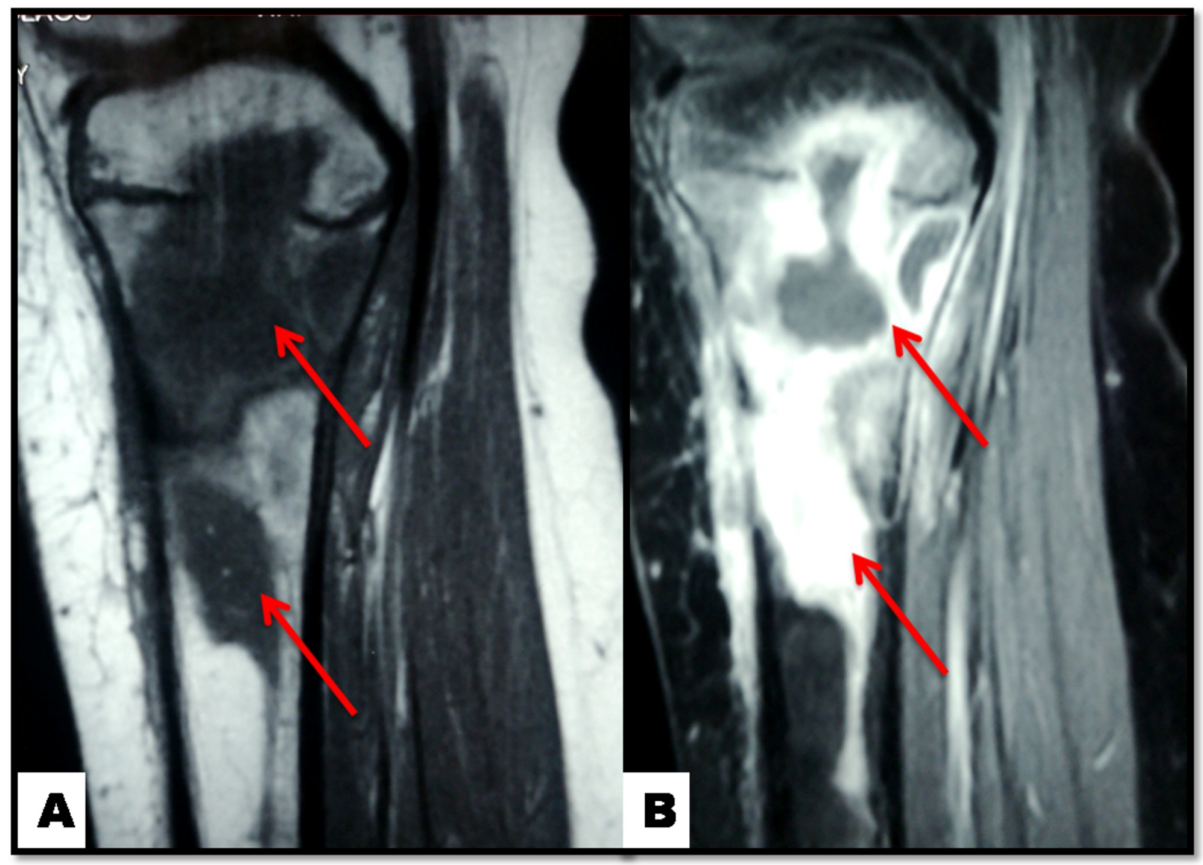

FIGURE 4: Knee MRI: osteomyelitis of the proximal tibia (red arrows)

A: sagittal T1-weighted sequence; B: sagittal T2-weighted sequence 


\section{Cureus}

were cultured onto selective agar media and inoculated in blood culture bottles. Traditional cultures remained negative. The cultures inoculated in blood culture bottles revealed Salmonella non-typhi sensitive in cephalosporins and quinolones. After PIDS consultation, IV ceftriaxone was administered for 12 days and two weeks per os antibiotic treatment (ciprofloxacin) was followed as an outpatient.

The patient was followed up by clinical, imaging and laboratory (WBC, ESR and CRP) examinations every week the first month, monthly the next five months and every six months until the two years follow up. At six month post-surgery follow up a knee MRI was performed (Figure 5). MRI revealed that the bone marrow had improved and the lytic lesion (Salmonella osteomyelitis) was reduced. At two years follow up the patient had fully recovered (Figure 6).

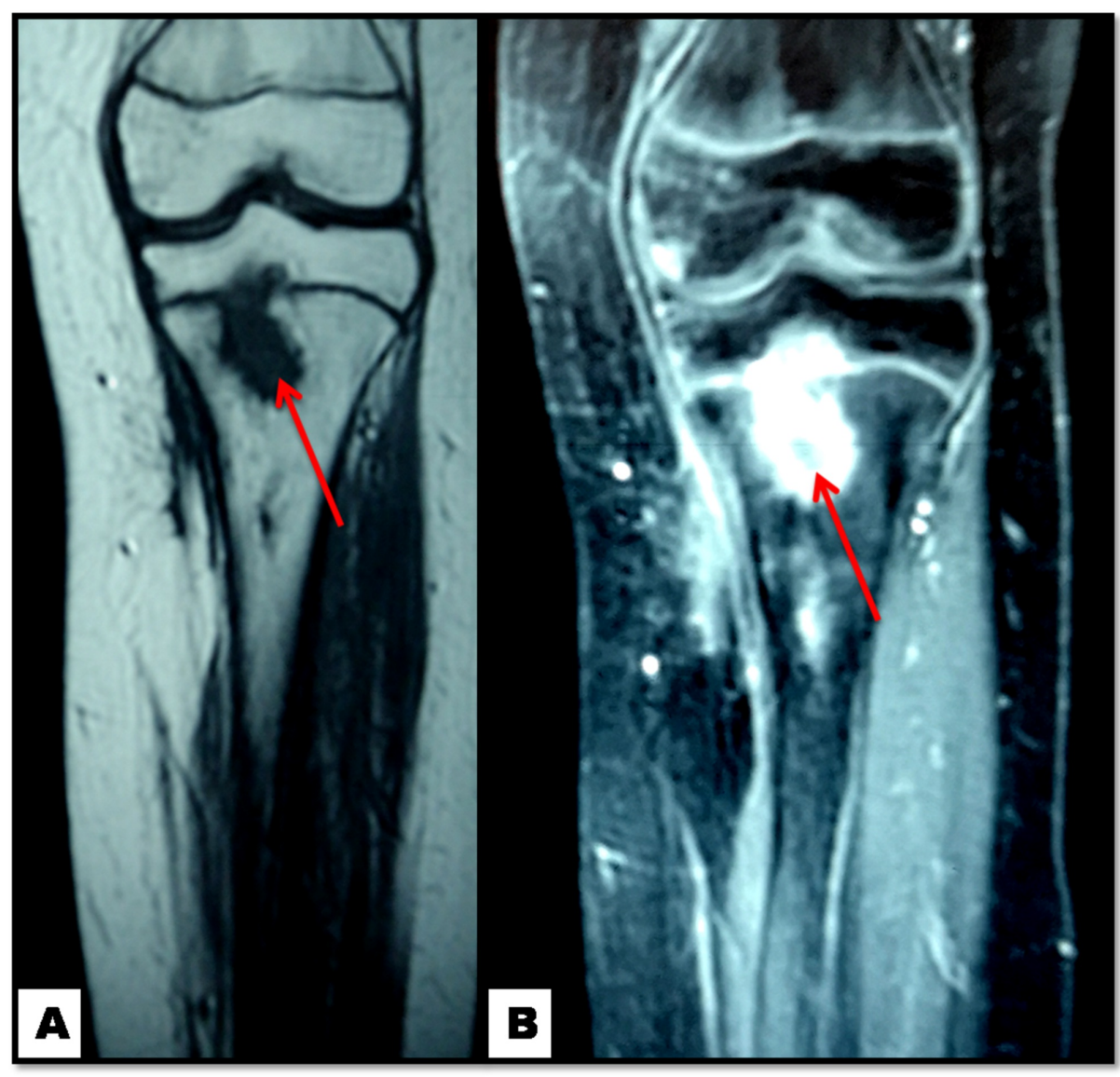

FIGURE 5: Knee MRI: osteomyelitis of the proximal tibia (red arrows) at six months follow up

A: coronal T1-weighted sequence; B: coronal T2-weighted sequence 


\section{Cureus}

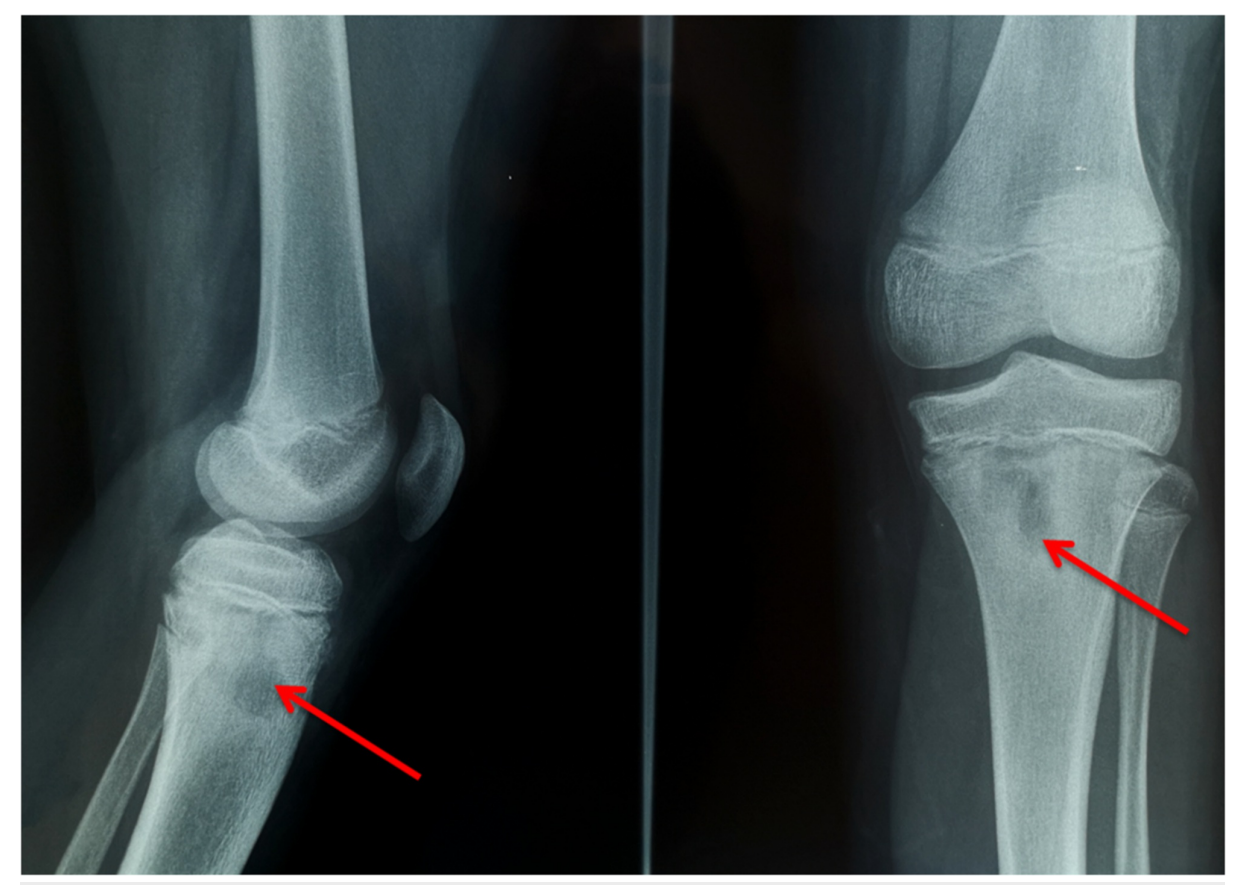

FIGURE 6: Knee radiographs at two years follow up: osteomyelitis of the proximal tibia (red arrows)

\section{Discussion}

In humans, Salmonella infection can manifest with variable clinical symptoms. It can be divided into five syndromes: gastroenteritis (food poising), enteric fever (typhoid), bacteremia or septicemia, focal infection (including soft tissue or bone infection) and a chronic carrier state [1,5].

Salmonella infection is one of the most common food-borne infections. It is caused by contaminated water, non-pasteurized milk and food (eggs, poultry) [1, 5-6]. Amphibians and cold-blood reptiles (like snakes, turtles and lizards) can cause Salmonella infections through skin contact (exotic pet reptile owners) or by use in folk medicine (snake-based medicines) [6].

Osteomyelitis is a known condition but a rare manifestation of Salmonella. Salmonella osteomyelitis is counting for $0.8 \%$ of all Salmonella infections and only $0.45 \%$ of all types of osteomyelitis [1-8]. The most common strains of Salmonella causing osteomyelitis in adults are Salmonella typhimurium, Salmonella enteritidis, Salmonella enterica subsp. arizonae, Salmonella typhi and Salmonella paratyphi [3-4]. Usually, Salmonella osteomyelitis is associated with hemoglobinopathies, such as sickle-cell disease or thalassemia and it seems to be a significant cause of morbidity and mortality in these individuals [1, 3-8]. In this population group, when a bone infection is presented, Salmonella osteomyelitis should be suspected [7].

Salmonella osteomyelitis is usually located in the diaphyseal region of long bones, typically in the femur and the humerus [3-4, 6]. Other bones commonly affected are the radius, ulna, tibia, and vertebrae [3]. Our case concerned the proximal tibia at the metaphyseal and epiphyseal region, which is uncommon. It was very difficult to diagnose because of the uncommon location of the lytic lesion and because our patient was a previously healthy child.

Clinical, radiological (with plain radiographs, CT scan, MRI scan) and laboratory evaluation should be provided in order to diagnose osteomyelitis. Salmonella osteomyelitis is difficult to diagnose. Clinical and radiological presents are indistinguishable from osteomyelitis caused by other microorganisms [1]. Signs of osteomyelitis caused by Salmonella are not revealed until 10 to 14 days after infection in plain radiographs and diagnosis is often delayed during the acute phase [1]. The duration of symptoms can range from a few months to several years [6]. In our case, the diagnosis of Salmonella osteomyelitis was delayed for three months.

Clinical deficits, radiographs, CT scan, MRI scan and inflammation markers are used to diagnose osteomyelitis. Blood cultures and cultures on agar media can identify the microorganism that causes osteomyelitis and the sensitivity in antibiotics, so the appropriate therapy is administered. In some cases though, it is difficult to identify the microorganisms in traditional cultures. In our case, Salmonella was isolated only when we inoculated sample in blood culture bottles. 
Targeted therapy for Salmonella osteomyelitis, according to the results of the culture, is fundamental. Usually, Salmonella is sensitive to penicillin antibiotics, third-generation cephalosporin, fluoroquinolones and chloramphenicol [1, 3-8]. Salmonella infection requires extensive and sometimes multiple debridements in addition to prolonged antibiotic therapy [6]. Duration of treatment ranges from five weeks to six months [4]. In our case, Salmonella non-typhi was sensitive in all the above antibiotics. Our patient required two surgical debridement procedures and two periods of antibiotic therapy.

\section{Conclusions}

The targeted treatment of Salmonella osteomyelitis is important and should be started as soon as possible. When traditional cultures are negative, inoculation of aspirates into blood culture bottles can be useful to diagnose Salmonella osteomyelitis in order to perform appropriate treatment.

\section{Additional Information}

\section{Disclosures}

Human subjects: Consent was obtained by all participants in this study. Conflicts of interest: In compliance with the ICMJE uniform disclosure form, all authors declare the following: Payment/services info: All authors have declared that no financial support was received from any organization for the submitted work. Financial relationships: All authors have declared that they have no financial relationships at present or within the previous three years with any organizations that might have an interest in the submitted work. Other relationships: All authors have declared that there are no other relationships or activities that could appear to have influenced the submitted work.

\section{References}

1. Zhan C, Du J, Chen L: Salmonella osteomyelitis in a previously healthy neonate: a case report and review of the literature. Ital J Pediatr. 2018, 44:28. 10.1186/s13052-018-0464-2

2. Dodwell ER: Osteomyelitis and septic arthritis in children: current concepts . Curr Opin Pediatr. 2013, 25:58-63. 10.1097/MOP.0b013e32835c2b42

3. McAnearney S, McCall D: Salmonella Osteomyelitis. Ulster Med J. 2015, 84:171-172.

4. Tsagris V, Vliora C, Mihelarakis I, Syridou G, Pasparakis D, Lebessi E, Tsolia M: Salmonella osteomyelitis in previously healthy children: report of 4 cases and review of the literature. Pediatr Infect Dis J. 2016, 35:116117.

5. Tonogai I, Hamada Y, Hibino N, Sato R, Henmi T, Sairyo K: Salmonella osteomyelitis of the distal radius in a healthy young adult patient: report of a rare case and literature review. J Med Invest. 2015, 62:97-99. $10.2152 /$ jmi.62.97

6. Salem KH: Salmonella osteomyelitis: a rare differential diagnosis in osteolytic lesions around the knee . J Infect Public Health. 2014, 7:66-69. 10.1016/j.jiph.2013.07.004

7. Adeyokunnu AA, Hendrickse RG: Salmonella osteomyelitis in childhood. A report of 63 cases seen in Nigerian children of whom 57 had sickle cell anaemia. Arch Dis Child. 1980, 55:175-184. 10.1136/adc.55.3.175

8. Sanchez AA, Mazurek MT, Clapper MF: Salmonella osteomyelitis presenting as fibrous dysplasia. A case report. Clin Orthop Relat Res. 1996, 330:185-189. 10.1097/00003086-199609000-00023 